\title{
Evaluation of spot urinary albumin-creatinine ratio as a screening tool in prediction of preeclampsia
}

\author{
Shaily Agarwal, Pratibha Devi, Renu Gupta, Pratima Verma, Apoorva Agarwal \\ Corresponding author: Dr. Pratima Verma, Assistant Professor, Department of Obstetrics and \\ Gynaecology, G.S.V.M Medical College, Kanpur, Uttar Pradesh, India; \\ Email : pratima2011@gmail.com
}

Distributed under Attribution-Non Commercial - Share Alike 4.0 International (CC BY-NC-SA 4.0)

\begin{abstract}
Objective: The prime objective of this study was to determine the effectiveness of preeclampsia prediction in asymptomatic cases with a spot urinary albumin creatinine ratio measured between 20 to 24 weeks of gestation. Materials and methods: The study included 156 women with a singleton pregnancy, normal renal function, and no evident proteinuria, attending antenatal clinics between 20 to 24 weeks of gestation at GSVM Medical College. Spot urine samples were taken between 20-24 weeks of gestation and urinary albumin was determined by the Biuret method and creatinine measured by modified Jaffe's method. Results: Preeclamptic patients who were asymptomatic in early pregnancy and developed preeclampsia, later on, yielded significantly higher spot UACR values than the normal ones. UACR value satisfactorily predicted preeclampsia well before the onset of clinical manifestations with high sensitivity and specificity. Especially, lower UACR values accurately corresponded to normotensive cases with a negative predictive value of $92 \%$. Out of 156 subjects, 24 developed preeclampsia. The sensitivity and specificity of UACR in predicting the development of preeclampsia were $62 \%$ and $84 \%$ respectively. Conclusion: The spot urine ACR test has the potential to predict preeclampsia and thereby reduce maternal and fetal mortality and morbidity by early intervention and intensive clinical follow-up. Thus it is an excellent predictor of preeclampsia.
\end{abstract}

Keywords: Urine albumin creatinine ratio, preeclampsia, screening, negative prediction value.

Preeclampsia is a disorder of pregnancy associated with new onset hypertension, which occurs mostly after 20 weeks of gestation and frequently near term. Preeclampsia is the second leading cause of maternal mortality ${ }^{1}$. It is dangerous for mother and baby, as well as unpredictable in onset and progression. Worldwide, the incidence of preeclampsia ranges between $2-8 \%$ of pregnancies. ${ }^{2}$ WHO estimates a higher incidence of this lethal disorder in developing countries (up to seven times) than in developed countries. ${ }^{3}$ As per the National Institute of Health and Family Welfare (NIHFW), the incidence of preeclampsia in India is reported to be $8-10 \%$ among pregnant women. ${ }^{4}$

It is a multiorgan disease process of unknown etiology characterized by de novo development of hypertension and proteinuria after 20 weeks of gestation. As per the new
ACOG Practice Bulletin, although hypertension and proteinuria were the classical criteria for the diagnosis of preeclampsia, the presence of any one of the following severe features in women with gestational hypertension without proteinuria should now also be classified as preeclampsia. These features include thrombocytopenia, impaired liver function test, severe persistent right upper quadrant or epigastric pain, renal insufficiency, pulmonary edema, new onset headache unresponsive to acetaminophen not suggestive of an alternative diagnosis. ${ }^{5}$ Studies for etiopathogenesis have proved that both alterations in the regulation and signaling of the angiogenesis pathway as well as endothelial dysfunction contribute to the inadequate cytotrophoblast invasion converting the usual low resistance high flow system into high resistance and low flow through

Received: $4^{\text {th }}$ April 2020, Peer review completed: $25^{\text {th }}$ February 2021, Accepted: $7^{\text {th }}$ March 2021.

Agarwal S, Devi P, Gupta R, Verma P, Agarwal A. Evaluation of spot urinary albumin-creatinine ratio as a screening tool in prediction of preeclampsia. The New Indian Journal of OBGYN. 2022; 8(2): 269 - 72. 
the placental vasculature. ${ }^{6}$ Endothelial dysfunction has been evident as early as 22 weeks of gestation by the demonstration of microalbuminuria by this time. The level of anti-angiogenic factors also starts rising as early as 17 weeks of gestation. $^{7}$

Preeclampsia has a dreadful impact on both maternal and fetal outcomes. It is not only the second leading cause of maternal mortality constituting $12 \%$ to $18 \%$ of pregnancy related maternal deaths but also a leading cause of fetal mortality and morbidity. ${ }^{1}$ The optimal way to minimize maternal and fetal complications is to prevent, diagnose and treat the disease. For prevention and timely diagnosis, we need screening and confirmatory tests to predict the disease before its clinical onset. Prediction of preeclampsia in the early stage of pregnancy can help prevent the onset of clinical features thus saving both the mother and fetus from the wrath of this disorder. The various available screening tools are a combination of maternal risk factors, uterine artery doppler, mean arterial pressure, maternal serum pregnancy associated plasma protein-A, and placental growth factor which can identify about $95 \%$ of cases of early onset preeclampsia with a false positive rate of $10 \%$. What remains unresolved is the use of a universally acceptable screening test that can predict preeclampsia before its clinical onset. Various studies have been done to identify such a test but the predictive ability of individual tests varies and none accurately predict the disease before the onset of clinical symptoms.

Persistent microalbuminuria indicates a high probability of damage to the glomerular filtration capacity of the kidney and is of great diagnostic relevance in pregnancy as a possible predictor of developing preeclampsia. For the quantitative evaluation of proteinuria in pregnancy measurement of protein excretion in 24 hours urine collection has been the longstanding "gold standard". However, 24 hours urine collection is time consuming, cumbersome, and less reliable because of the difficulty in collecting the sample correctly. So to overcome this drawback measurement of urinary albumin to creatinine ratio (UACR) in spot urine samples is undertaken. This is based on the logistics that with a non fluctuating glomerular filtration rate, urinary creatinine is excreted at a fairly constant rate, thus making it useful as an internal reference. It avoids the influence of variations in urinary solute concentration and provides a more convenient and rapid method to assess protein excretion for the prediction of preeclampsia.
So, the present study attempts to use a single spot urinary albumin creatinine ratio to detect the predictive value of urinary albumin to creatinine ratio in pregnant women who are free from symptoms of preeclampsia. This test is more feasible in clinical practice as a screening test than the 24 hours collection method which is complicated as well as time consuming. Maternal and fetal outcome in patients with normal and raised spot urinary albumin creatinine ratio (UACR) was assessed and studied as well.

\section{Material and methods}

This was a prospective observational study of 180 women attending an antenatal clinic for routine antenatal care, between 20 to 24 weeks gestation in the department of obstetrics and gynaecology, Upper India Sugar Exchange Maternity Hospital, GSVM Medical College, Kanpur, and other associated hospitals from December 2017 to August 2019. The study was approved by the ethical committee GSVM medical college, Kanpur.

Inclusion criteria: Singleton pregnancy, gestational age of 20-24 weeks by last menstrual period (LMP) and verified by ultrasonography (USG), normal renal function, no proteinuria, dipstick negative.

Exclusion criteria: Multifetal pregnancy, gestational age $<20$ weeks and $>24$ weeks, dipstick positive, ongoing urinary tract infection (UTI), women with new onset headache unresponsive to medication, acute renal disease, chronic renal disease, gestational diabetes, pre-existing diabetes, known major fetal abnormalities, or fetal demise, pregnancy after assisted reproduction technology.

At the time of registration, after taking informed consent, detailed data was collected. Age, parity, weight, body mass index (BMI), address, occupation, socioeconomic status, smoking or tobacco intake, alcohol intake, history of past illness, family history of preeclampsia, history of pregestational diabetes, history of chronic hypertension or renal diseases per inclusion and exclusion criteria. In this study total, 1000 patients were included, out of 1000 patients, 180 patients were fulfilling the inclusion and exclusion criteria and registered for this study. Spot urine sample of the registered patient was taken in the next visit. Venous blood $(10 \mathrm{ml})$ was collected into a test tube for routine antenatal investigation. Glucose tolerance test, urine routine microscopy and urine culture sensitivity, kidney function test, liver function test was also done to rule out diabetes, kidney disease, and liver disease. General, systemic and obstetric examinations were carried out on the same day. In the study, a total of 180 cases were registered out of which 156 cases were analyzed and cases lost to follow up were 24 . 
Spot urine sample of the registered patients was taken between 20-24 weeks of gestation. A clean catch spot urine sample $(5 \mathrm{ml})$ of the subjects, was collected in a clean sterile container. Urinary albumin was determined by the Biuret method and creatinine was measured by modified Jaffe's method. The resulting UACR value for each case was carefully noted down. The patients were put under regular follow up at 28 weeks, 32 weeks, 34 weeks, 36 weeks then weekly until delivery. A specific note was made for the development of preeclampsia during the antenatal period and/or at the time of delivery. For the prediction of preeclampsia, a UACR of 0.2 is taken as the cutoff value.

The statistical analysis of the data obtained in the present study was carried out using the statistical package for the social sciences (SPSS version 20). Pearson coefficient ( $p$ value) was calculated by Students' unpaired t-test. A p-value of less than 0.0001 was considered statistically significant.

\section{Result}

In the study, a total of 180 cases were registered out of which 156 cases were analyzed and cases lost to follow up were 24 . The mean age of the patients included in our study was $25.6 \pm 3.21$ years. Sixty two (39.74\%) were primipara and $94(60.26 \%)$ were multipara patients.

Table 2: Statistical values for normotensive and preeclamptic cases

\begin{tabular}{lll}
\hline Table 1: Association of UACR with preeclampsia & \\
\hline UACR & Normotensive & Preeclampsia \\
\hline Test positive $(>0.2)$ & $20(12.8 \%)$ (False Positive) & $15(9.6 \%)$ (True \\
Test negative $(<0.2)$ & $112(71.8 \%)$ (True Negative) & $9(5.8 \%)$ False N \\
\hline Total & $132(84.6 \%)$ & $24(15.4 \%)$ \\
\hline Parameters & Normotensive (132) & Preeclamptic (24) \\
\hline Mean & 0.1 & 0.28 \\
Median & 0.07 & 0.23 \\
Range & 0.004 to 1.5 & 0.06 to 0.49 \\
Standard deviation & $+/-0.14$ & $+/-0.45$ \\
Mean difference & 0.18 & \\
P - value & $<0.0001$ & \\
\hline
\end{tabular}

\begin{tabular}{ll}
\multicolumn{2}{l}{ Table 3: Accuracy of preeclampsia prediction with UACR cut-off of $\mathbf{0 . 2}$} \\
\hline Sensitivity & $62 \%$ \\
Specificity & $84 \%$ \\
Positive predictive value & $56 \%$ \\
Negative predictive value & $92 \%$ \\
Relative risk & 5.762 \\
P-Value & $<0.0001$ \\
\hline
\end{tabular}

Out of 156 women, 35 showed a UACR measurement of more than 0.2 , which is the cut-off value taken for preeclampsia prediction, whereas the incidence of preeclampsia in our study group came out to be $15.4 \%$ i.e, 24 patients (table 1). Statistical results from table 1 are shown in table 2 and table 3 . Out of 24 preeclamptic patients, 5 patients developed severe preeclampsia and 19 patients were non-severe preeclampsia. 2 out of 24 preeclamptic patients developed HELLP syndrome. Fortunately, none of the patients in our study developed eclampsia.

\section{Discussion}

The core finding of the study shows that the spot urinary albumin creatinine ratio at 20-24 weeks was significantly higher in women who subsequently developed preeclampsia with values more than 0.2 . Normotensive women were more likely to have UACR values less than 0.2. Upadhyay A, Dayal $\mathrm{M}$ and Fatema $\mathrm{K}$ et al also took cut-off UACR value at 0.2 and reached the same conclusion. ${ }^{8,9}$

The incidence of preeclampsia in our study group came out to be $15.8 \%$ which is comparable to the incidence in developing countries which is $4-18 \%$. Mishra VV et al showed a comparable rate of preeclamptic incidence at $12.8 \%$ in the sample size of 62 women. ${ }^{1}$

Table no. 3 shows that the sensitivity of UACR at 0.2 as a screening test to predict preeclampsia in our study was found to be $62 \%$, which is comparable to the studies done by Fatema K et al (50\%-68\%) ${ }^{9}$ and Gupta $\mathrm{N}$ et al $(67 \%) .{ }^{10}$ Specificity $(84 \%)$, positive predictive value $(56 \%)$, and negative predictive value(92\%) derived in our study were quite high and comparable to earlier studies by Upadhyay A, Dayal M. ${ }^{8}$

The negative predictive value is $92 \%$ in this study which is comparable to Fatema $\mathrm{K}$ et al i.e $96.42 \%{ }^{9}$, Upadhyay A, Dayal $M$ i.e $97.5 \%{ }^{8}$ and Oya Demirci i.e $91.2 \%{ }^{11}$, which showed that if NPV is high then patients had less chance of developing preeclampsia.

\section{Conclusion}

Spot UACR ratio is higher in asymptomatic women who developed preeclampsia later on. When measured early in the second trimester, UACR more than 0.2 predicted preeclampsia well before the onset of clinical manifestation of preeclampsia with the sensitivity of the test being $62 \%$, specificity being $84 \%$, the positive predictive value of $56 \%$, and negative predictive value of $92 \%$. So spot UACR can be used as a good screening tool for the prediction of preeclampsia in early pregnancy.

Measurement of protein excretion in a $24 \mathrm{hr}$. urine collection has been the long standing gold standard for the quantitative evaluation of proteinuria in pregnancy however it is time consuming, inconvenient, and not always reliable because of difficulty in collecting the sample correctly. Measurement of UACR in a spot urine sample is a more convenient and rapid method to assess protein excretion for prediction of preeclampsia. However, more studies are 
The New Indian Journal of OBGYN. 2021 (January-June);8(2)

required with a larger sample size to establish its role in correctly predicting preeclampsia.

\section{Conflict of interest: None. Disclaimer: Nil.}

\section{References}

1. Mishra VV, Goyal PA, Priyankur R, Choudhary S, Aggarwal RS, Gandhi K, Vyas B, Hokabaj S. Evaluation of Spot Urinary Albumin-Creatinine Ratio as Screening Tool in Prediction of Pre-eclampsia in Early Pregnancy. The Journal of Obstetrics and Gynecology of India. 2017 Dec 1; 67(6): 405-8.

2. Steegers EA, Von Dadelszen P, Duvekot JJ, Pijnenborg R. Pre-eclampsia. The Lancet. 2010 Aug 21; 376(9741): 631-44.

3. Kooffreh ME, Ekott M, Ekpoudom DO. The prevalence of pre-eclampsia among pregnant women in the University of Calabar Teaching Hospital, Calabar. Saudi Journal of Health Sciences. 2014; 3(3):133-6.

4. National Health portal. National Institute of Health and Family Welfare. Pre-eclampsia. [Cited on 18 February 2018]. Available on: https://www.nhp.gov. in/ disease/ gynaecology-and-obstetrics/ preeclampsia.

5. Hypertension G. Preeclampsia. ACOG Practice Bulletin No. 202. American College of Obstetricians and Gynecologists. Obstet Gynecol. 2019;133(1): e1-25.

6. Redman CWG, Sargent IL, Roberts JM. Immunology of abnormal pregnancy and preeclampsia. In: Lindheimer MD, Roberts JM, Cunningham FG, editors. Chesley's Hypertensive Disorders of Pregnancy. $3^{\text {rd }}$ ed. New York: Elsevier; 2009. p.129.

7. Young BC, Levine RJ, Karumanchi SA. Pathogenesis of preeclampsia. Annual Review of Pathology. 2010 Feb 28; 5: 173-92.
8. Upadhyay A, Dayal M. Screening for preeclampsia by urine albumin to creatinine ratio. The New Indian Journal of OBGYN. 2018; 4(2): 117 - 20.

9. Fatema K, Khatun M, Akter S, Ali L. Role of urinary albumin in the prediction of preeclampsia. Faridpur Medical College Journal. 2011; 6(1): 14-8.

10. Gupta N, Gupta T, Asthana D. Prediction of preeclampsia in early pregnancy by estimating the spot urinary albumin/creatinine ratio. The Journal of Obstetrics and Gynecology of India. 2017 Aug 1; 67(4): 258-62.

11. Demirci O, Kumru P, Arınkan A, Ardıç C, Arısoy R, Tozkır E, et al. Spot protein/creatinine ratio in preeclampsia as an alternative for 24-hour urine protein. Balkan Medical Journal. 2015 Jan; 32(1): 51-5.

\section{Shaily Agarwal ${ }^{1}$, Pratibha Devi ${ }^{2}$, Renu Gupta ${ }^{3}$, Pratima Verma $^{4}$, Apoorva Agarwal ${ }^{5}$}

${ }^{1}$ Associate Professor, Department of Obstetrics and Gynaecology, G.S.V.M Medical College, Kanpur, Uttar Pradesh, India; ${ }^{2}$ Senior Resident, Department of Obstetrics and Gynaecology, G.S.V.M Medical College, Kanpur, Uttar Pradesh, India; ${ }^{3}$ Professor, Department of Obstetrics and Gynaecology, G.S.V.M Medical College, Kanpur, Uttar Pradesh, India. (attached GMC Kannauj); ${ }^{4}$ Assistant Professor, Department of Obstetrics and Gynaecology, G.S.V.M Medical College, Kanpur, Uttar Pradesh, India; ${ }^{5}$ Professor and Head, Department of Anaesthesiology, Critical care and pain management, GMC Jalaun, Uttar Pradesh, India. 